\title{
The Analysis of Temperature, Depth, Salinity Effect on Acoustic Speed for a Vertical Water Column
}

\author{
Anisur Rahman, Vallipuram Muthukkumarasamy, Elankayer Sithirasenan \\ School of Information and Communication Technology, Griffith University \\ Gold Coast, QLD 4222, Australia \\ \{anis.rahman, v.muthu, e.sithirasenan\}@griffith.edu.au
}

\begin{abstract}
This paper studies and analyzes the influence of temperature, depth and salinity on speed of acoustic signal for a vertical water column. In underwater wireless sensor networks (UWSN), determination of precise acoustic signal is sometimes necessary to determine the coordinates of sensors for the validity of data. The effect of temperature on sound speed is predominant where temperature change is noticeable. In the deep ocean where temperature change is very nominal, depth (pressure) becomes predominant and salinity effect is even less. In this paper we have shown how these variables affect acoustic signal speed for a specific depth of water column where temperature, depth and salinity changes are linear.
\end{abstract}

Keywords-underwater; acoustic signal speed; temperature; depth; salinity

\section{INTRODUCTION}

Oceans cover vast areas and hold significant explored and unexplored resources that provide a great scope for the exploration and monitoring. As wireless sensor networks (WSNs) excel at monitoring terrestrial environments, there is a natural tendency to try and apply them to oceanic environments. The resulting challenges spawn the field of underwater sensor networks.

The knowledge of location of a node in the network not only improve the operation of the network (e.g. geographical routing), but also add significance to the data collected by the nodes to enable applications for oceanographic data collection, pollution monitoring, offshore exploration, disaster prevention, assisted navigation and tactical surveillance applications [1]. Despite varieties of application of UWSN, idea of submerged wireless communication may still seem far-fetched and got attention of researchers since last decades. As positions of submerged sensors plays a vital role for the significance of validity of data, so determining the coordinate precisely is very crucial. Most distance measurement techniques considers speed of acoustic signal constant, usually $1500 \mathrm{~m} / \mathrm{s}$. However, several studies shows that speed of acoustic signal varies with temperature, depth (pressure), and salinity [2]. Hence, the constant speed assumption incorporates an error into the localization error.

However, in the recent years, there has been a growing interest to explore and fulfil the needs of a multitude of underwater applications. So, it is crucial to provide accurate environmental data using underwater sensors, to provide and maintain sanctuary for the marine life. In addition to underwater sensors, the network may also comprise surface stations and autonomous underwater vehicles and regardless of the type of deployment (outdoor, indoor, underground or underwater), the location of sensors needs to be determined for meaningful interpretation of the sensed data [3].

\section{PROBlem Domain}

In the proposed method a beacon (boat or buoy) would be at the surface of water column and few sensors that require the distance measurement would be deployed. It is understood that the sensor nodes could be in any layers as long as the variables satisfies constrains of Mackenzie equation. To determine the average acoustic speed, it is required to know the temperature, depth and salinity of water at the beacon and sensors position. To know these variables of the beacon is somewhat strait forward, i.e., temperature and salinity of the water surface can be determined at depth zero. However, to know those variables for sensor nodes is challenging if particular sensors (i.e., temperature, pressure and salinity sensors) are not incorporated with the nodes. In this paper, we will show the effect of these variables on acoustic speed for a shallow water column.

For the sake of simplicity, at present we assume that the submerged sensors are static for the time of computation - the mobility of the sensor nodes are not considered here because our focus is to determine average speed and to analyse the effect of aforesaid variables on speed. A solvable configuration of one beacon with multiple submerged sensors is denoted in Fig. 1. We have simulated a water column of $200 \mathrm{~m}$ in depth; because most of the marine explorations take place on the shallow water.

\section{Determination of AVERAge SpeEd of Acoustic Signal}

A typical speed of acoustic waves near the ocean surface is about $1500 \mathrm{~m} / \mathrm{s}$, more than four times faster than the speed of sound in air. However, the speed of sound in underwater is affected prominently by temperature, depth and salinity. As these parameters are variable and different from place to place in the water; so as the speed. And the speed of sound $v$ in water can be calculated according to the Mackenzie equation [4]. 


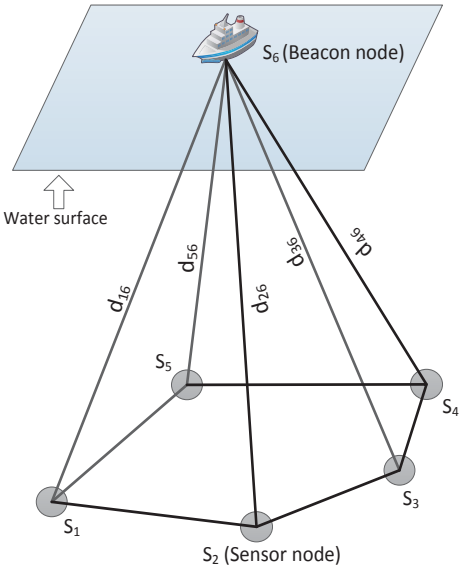

Fig. 1. Subset composed of one beacon and submerged sensors

$$
\begin{aligned}
& v=1448.96+4.591 \mathrm{~T}-5.304 \times 10^{-2} \mathrm{~T}^{2}+2.374 \times 10^{-4} \mathrm{~T}^{3}+ \\
& 1.340(\mathrm{~S}-35)+1.630 \times 10^{-2} \mathrm{D}+1.675 \times 10^{-7} \mathrm{D}^{2}-1.025 \times 10^{-2} \\
& \mathrm{~T}(\mathrm{~S}-35)-7.139 \times 10^{-13} \mathrm{TD}^{3}
\end{aligned}
$$

where $\mathrm{T}, \mathrm{D}$ and $\mathrm{S}$ are temperature, depth and salinity and constrains for these variables are $2-30^{\circ} \mathrm{C}, 0-8000 \mathrm{~m}$ and $25-40 \mathrm{ppt}$ respectively. Mackenzie equation gives sound speed for any specific value of $\mathrm{T}, \mathrm{D}$ and $\mathrm{S}$ whereas we propose to determine average speed of sound through the water column. As the variation of salinity is not significant in different level of the water column except at the estuary, if we consider salinity (35ppt) to be a constant for any particular water column. The acoustic speed profile can be seen as in Fig. 2.

The top part of the ocean is called the surface layer, where temperature change is less significant than the layer beneath it, called thermocline that spreads over few hundred meters sometimes. In (1), it can be seen that temperature is the prominent factors that affects acoustic signal speed more than the others. In section IV simulation results suggest salinity has very minimal effect on speed of sound; moreover the change in salinity for a $200 \mathrm{~m}$ water column is not that significant.

Once the temperature, depth of the water column and salinity are measured and communicated with beacon, equipped with all the variables of top and bottom surface, we can compute the average speed of acoustic signal following (2).

$$
\begin{aligned}
& \bar{v}=f_{\text {avg }}(T, D, S)=\frac{1}{A} \iiint_{R} f(T, D, S) d A \\
& =\frac{1}{A} \int_{S_{i} \geq 25} \int_{D_{i} \geq 0} \int_{T_{i} \geq 2} f(T, D, S) d T d D d S
\end{aligned}
$$

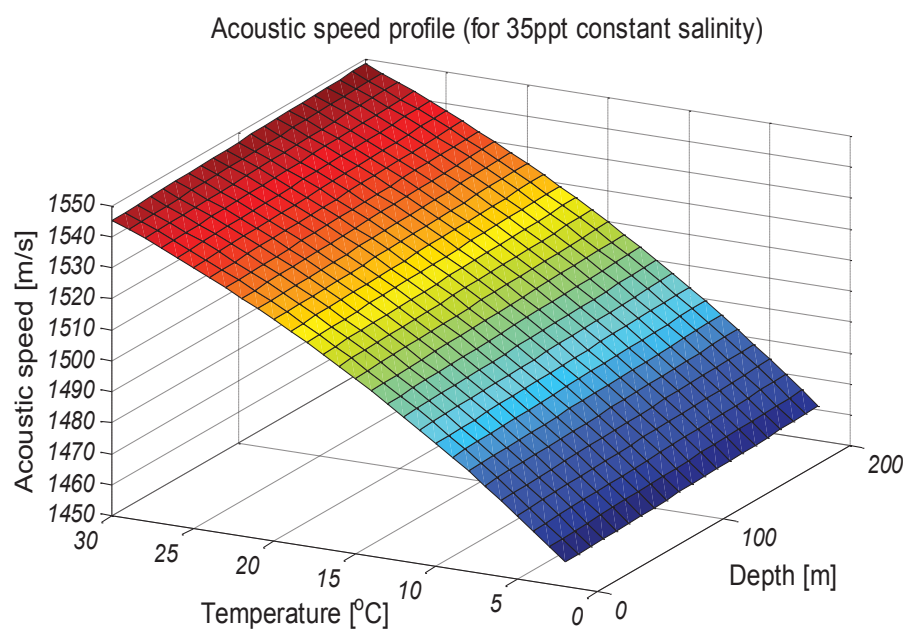

Fig. 2. Acoustic speed profile according to Mackenzie equation

here, 'A' is the area created by the limit of T, D, and S; $f(T, D, S)$ is the Mackenzie equation as in (1).

\section{SIMULATION RESULTS AND DISCUSSIONS}

In order to analyze the effect of temperature, depth and salinity on acoustic speed, we have simulated the method using Matlab. Average sound speed is computed for $200 \mathrm{~m}$ water column with $20^{\circ} \mathrm{C}$ surface and $10^{\circ} \mathrm{C}$ bottom temperature having salinity variation of $0.5 \mathrm{ppt}$ in Fig. 3. Gaussian noise has been added to $10^{\circ} \mathrm{C}$ bottom temperature. After 100 iterations the mean of 'average sound speed' is found to be $1507.6 \mathrm{~m} / \mathrm{s}$.

In Fig. 4 the effect of temperature on average sound speed has been portrayed. Average sound speed increases $7.3 \mathrm{~m} / \mathrm{s}$ due to change in surface temperature from $20^{\circ} \mathrm{C}$ to $25^{\circ} \mathrm{C}$ while bottom temperature is $10^{\circ} \mathrm{C}$. For $20^{\circ} \mathrm{C}$ surface temperature, average sound speed increases $1.7 \mathrm{~m} / \mathrm{s}$ for only $1^{\circ} \mathrm{C}$ increase in bottom temperature. $0.53 \%$ change due to $5^{\circ} \mathrm{C}$ change at the bottom temperature is quite significant.

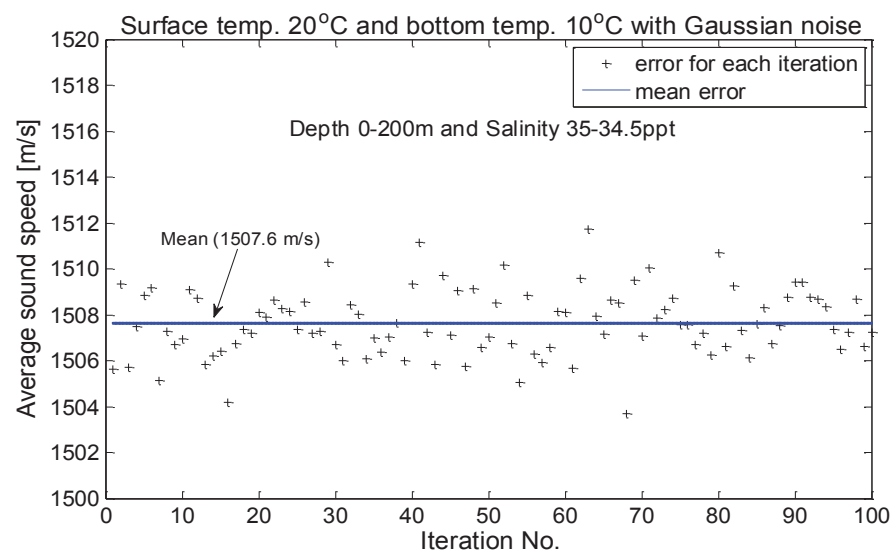

Fig. 3. Average speed variation due to additive Gaussian noise to bottom temp. 


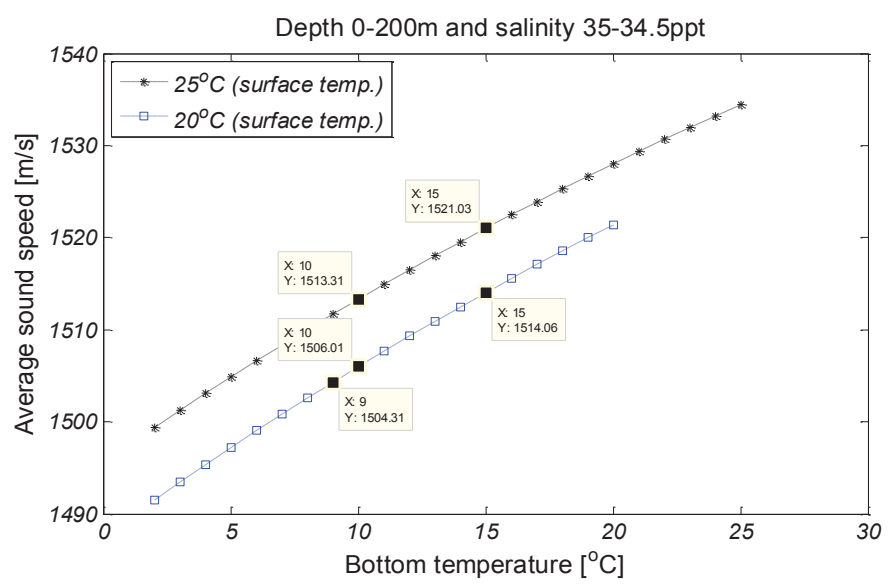

Fig. 4. Average speed variation due to temperature change

Sound speed change due to depth is not as significant as temperature which can be seen in Fig. 5. Average sound speed increases only $0.09 \mathrm{~m} / \mathrm{s}$ due to $10 \mathrm{~m}$ increase in water column for the same condition as in Fig. 4. So, for 200m water column, 5\% error in depth measurement can affect the average sound speed by $0.059 \%$ which is almost negligible.

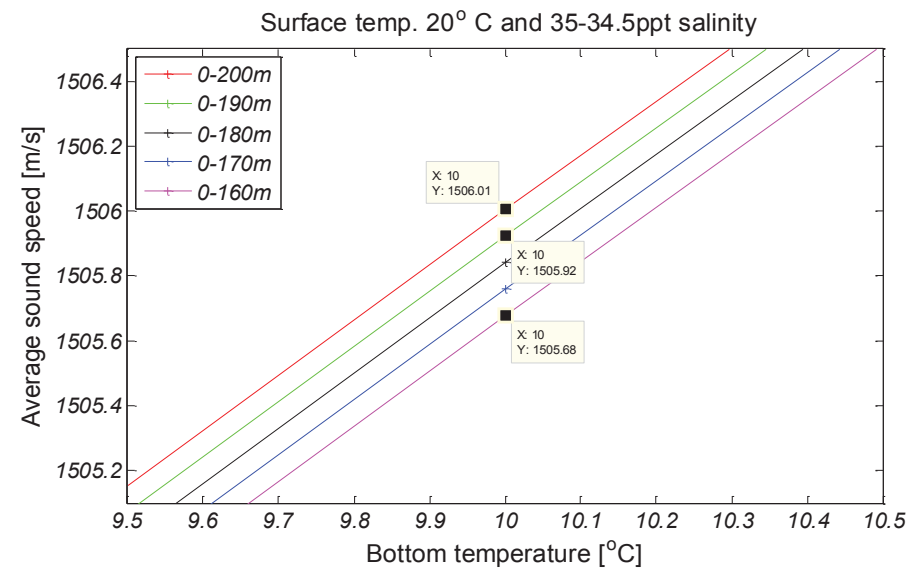

Fig. 5. Average speed variation due to depth change

Fig. 6 suggests salinity increase affects average speed of sound positively; $0.14 \mathrm{~m} / \mathrm{s}$ increase of average sound speed due to $0.25 \mathrm{ppt}$ salinity change at the bottom surface. $0.029 \%$ increase in average sound speed for $0.75 \mathrm{ppt}$ salinity change at the bottom surface is not quite dramatic as it is the case in temperature change.

From the above simulation results, it can be inferred that temperature is the prime factor to be considered to avoid possible errors in average sound speed for any shallow water column. In other words, errors in temperature measurements will affect more than depth and salinity in calculation of the average sound speed that eventually affect the determination of coordinates of underwater sensor nodes.

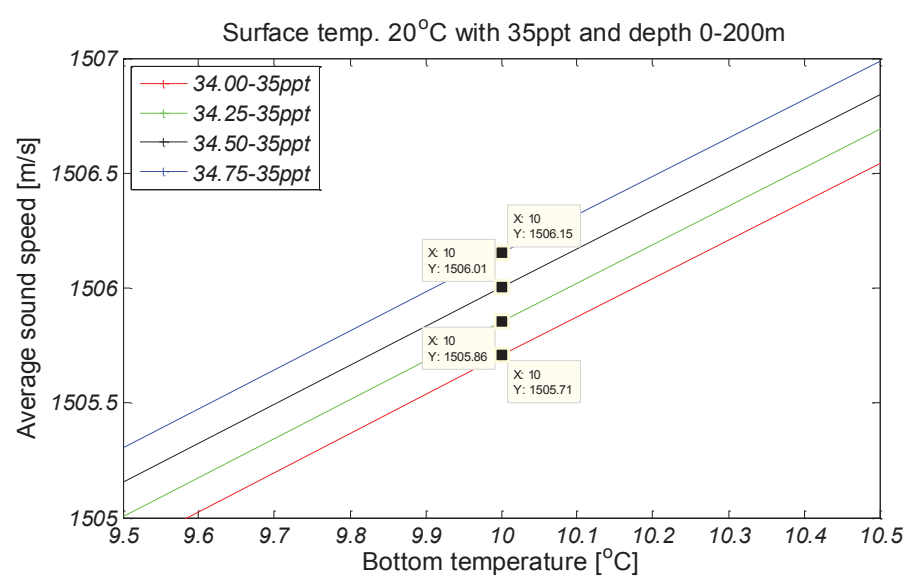

Fig. 6. Average speed variation due to salinity change

\section{CONCLUSIONS}

In this paper we have shown the effect of temperature, depth and salinity on average speed of acoustic signal for a vertical water column. The effect due to temperature change is quite significant whereas depth and salinity has minimal effect. The average sound speed is $1506.01 \mathrm{~m} / \mathrm{s}$ for the aforesaid conditions; however, the mean of 'average sound speed' with additive Gaussian noise to the $10^{\circ} \mathrm{C}$ bottom temperature for 100 iterations is found to be $1507.6 \mathrm{~m} / \mathrm{s}$. Hence, to get an accurate average sound speed for a shallow water column, temperature measurement error should be kept minimal for determining the exact coordinates of the sensors.

\section{REFERENCES}

[1] I. F. Akyildiz, D. Pompili, and T. Melodia, "Underwater acoustic sensor networks: research challenges," Ad Hoc Networks, vol. 3, pp. 257-279, 2005.

[2] C. T. Chen and F. J. Millero, "Speed of sound in seawater at high pressures," The Journal of the Acoustical Society of America, vol. 62, p. $1129,1977$.

[3] H. P. Tan, R. Diamant, W. K. G. Seah, and M. Waldmeyer, "A survey of techniques and challenges in underwater localization," Ocean Engineering, vol. 38 , pp. 1663-1676, 2011.

[4] K. V. Mackenzie, "Nine - term equation for sound speed in the oceans," The Journal of the Acoustical Society of America, vol. 70, p. 807, 1981. 